\title{
WNN Fault Diagnosis Model with Modified Parameter Initialization for Analog Circuit
}

\author{
Yanming Wei ${ }^{1, a}$, Baohua Zhang ${ }^{2, b}$, Jingjuan Sun ${ }^{2, c}$ \\ ${ }^{1}$ Xijing College, Xi’an, Shaanxi, 710123, China \\ ${ }^{2}$ Air Traffic Control and Navigation College, Air Force Engineering, University, Xi’an, Shaanxi, 710051, \\ China \\ aganxusheng123@163.com, bzbh888@sina.com, csunjj222@163.com
}

Keywords: Wavelet neural network; Parameter initialization; Analog circuit; Fault diagnosis

\begin{abstract}
To diagnose the fault in analog circuit correctly, a Wavelet Neural Network (WNN) method is proposed with improvement of parameter initialization. Considering the shortcoming of traditional randomization method of WNN initialization, a new initialization method is introduced to improve the capability of WNN model, which considers not only the initialization of connection weights, but also the initialization of the expansion factor and translation factor. The simulation shows that the proposed method has a good diagnosis capability for the fault in analog circuit.
\end{abstract}

\section{Introduction}

Information processing technology has developed rapidly in today, and circuits in electronic devices have become increasingly complex. With this, it has become increasingly difficult to effectively deal with equipment faults caused by analog circuits. Especially, with the improvement of circuit integration, the soft faults induced by the inherent tolerance of components can easily result in the deterioration of circuit and system performance, which brings large challenges to quickly locate and handle tolerance faults. Obviously, the conventional fault diagnosis method cannot satisfy the requirement, and the research of new diagnostic technology is imminent.

In 1962, Berkowitz proposed the resolvable concept of analog circuits, which began the initial research stage of fault diagnosis for analog circuit [1]. Navid and Willson demonstrated the sufficient conditions for the solvability of analog circuit network [2], which laid the theoretical foundation for fault diagnosis of analog circuit, and then the researchers have proposed a number of diagnosis methods, including fault dictionary method, parameter identification method, fault verification method, approximation method and so on [3][4]. Fault dictionary method is the most widely used and most practical method in fault diagnosis for analog circuit. Its advantage is easy to implement, but the shortcomings are also obvious.

Learning from the optimization experience of neural network, we introduce a WNN fault diagnosis method in analog circuit. By the case study of simulation, the effect and feasibility of the method were proved.

\section{Wavelet neural network}

WNN, which is a new feed-forward neural network developed on the basis of the wavelet analysis, is the result of organically integrating the wavelet analysis with the neural network [5]. In the paper, WNN uses the typical three layer structure, as shown in Fig. 1.

In the structure, $z_{i}(i=1, \cdots, I)$ is the input of network; $y_{t}(t=1, \cdots, T)$ is the output of network; $w_{i j}(i=1, \cdots, I ; j=1, \cdots, J)$ is the connection weight between input layer and hidden layer; $w_{0 j}(j=$ $1, \cdots, J)$ is the threshold of hidden layer node; $v_{j t}(j=1, \cdots, J ; t=1, \cdots, T)$ is the connection weight between hidden layer and output layer; $v_{0 t}(t=1, \cdots, T)$ is the threshold of output layer node. $I$ is the 
number of input nodes; $J$ is the number of hidden nodes; $T$ is the number of output nodes. $\psi_{a, b}(\cdot)$ is wavelet basis function of hidden layer. In the paper, Morlet wavelet function $\psi_{a, b}(t)$ $=\cos (1.75 t) e^{-t^{2} / 2}$ is adopted, where $a, b$ are the expansion factor and translation factor respectively. $f(\cdot)$ is the activation function of output layer which can be usually taken as Sigmoid function: $f(t)=1 /\left(1+e^{-t}\right)$. Then WNN model can be expressed as

$$
y_{t}^{l}=f\left[\sum_{j=0}^{J} v_{j t} \psi_{a, b}\left(\frac{\sum_{i=0}^{I} w_{i j} z_{i}^{l}-b_{j}}{a_{j}}\right)\right],(t=1, \cdots, T ; \quad l=1, \cdots, L)
$$

where $L$ is the total number of samples.

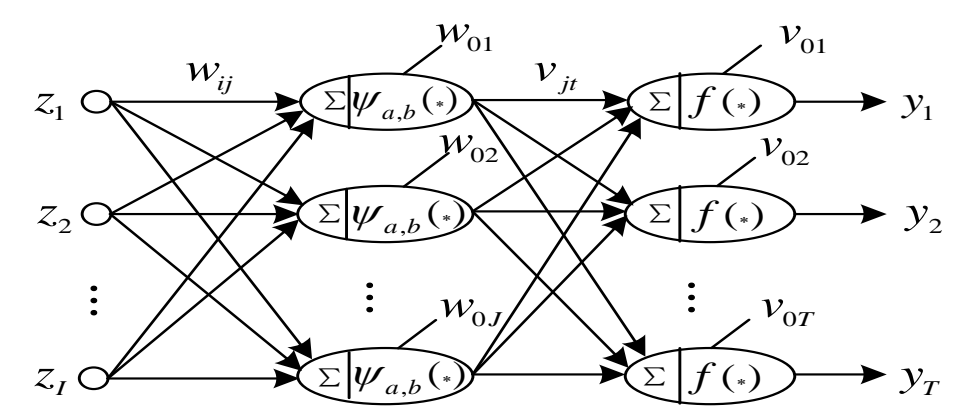

Input layer Hidden layer Output layer

Fig. 1 Structure of WNN

\section{Parameter Initialization}

Parameter initialization is an important part in WNN modeling. Selecting appropriate initial value of parameters can speed up the convergence rate; otherwise, the number of learning increases significantly, or even the algorithm is not convergence. In the past, the randomization method was adopted, obviously, it was not a scientific and effective initialization method. How to find the appropriate parameter initialization method through many parameters of WNN is an important issue to be solved [6].

Initializing network parameters, should consider not only the initialization of connection weights, but also the initialization of the expansion factor $a$ and translation factor $b$. Since the wavelet function has localized characteristics, if $a$ is too small, the wavelet basis function is too localized; if $b$ is chosen improperly, it may be away from the study range. Usually, the following empirical formula can be used to initialize the parameters.

For the connection weight $w_{i j}(i=1, \cdots, I ; j=1, \cdots, J)$ and $v_{j t}(j=1, \cdots, J ; t=1, \cdots, T)$, the random numbers with uniform distribute in $[-1,1]$ can firstly be taken as the initial values $w_{j i}^{\prime}$, and then the initial weights can be obtained by following empirical formula

$$
w_{i j}=w_{i j}^{\prime} /\left(d \sqrt{\sum_{i}^{I} w_{i j}^{\prime 2}}\right)
$$

where $d=\left(X_{\max }-X_{\min }\right) / 2$ denotes the center value of data; the sample maximum is $X_{\max }=$ $\max (X)$; the sample minimum is $X_{\min }=\min (X)$. The initialization process of $v_{j t}(j=1, \cdots, J ; t=$ $1, \cdots, T)$ is the same as it.

After setting initial weights, the expansion factor $a$ and translation factor $b$ in wavelet also need be initialized. According to the actual situation of fault diagnosis in analog circuit, we only consider the 
case that the number of input nodes is larger than 1. It can be found that, if the centre of time domain of mother wavelet is $t^{*}$ and the radius is $\Delta \psi$, then the wavelet expansion system is $\left[b+a t^{*}-\right.$ $\left.a \Delta \psi, b+a t^{*}+a \Delta \psi\right]$ in the concentrated region of time domain. To make the wavelet expansion system cover the entire range of the input vector, the initial setting of expansion factor and translation factor needs to satisfy the following formula:

$$
\begin{gathered}
\left\{\begin{array}{l}
b_{j}+a_{j} t^{*}-a_{j} \Delta \psi=\sum_{i=1}^{I} w_{j i} x_{\min } \\
b_{j}+a_{j} t^{*}+a_{j} \Delta \psi=\sum_{i=1}^{I} w_{j i} x_{\max }
\end{array}\right. \\
\left\{\begin{array}{l}
a_{j}=\frac{\sum_{i=1}^{I} w_{j i} x_{\max }-\sum_{i=1}^{I} w_{j i} x_{\min }}{2 \Delta \psi} \\
b_{j}=\frac{\sum_{i=1}^{I} w_{j i} x_{\max }\left(\Delta \psi-t^{*}\right)-\sum_{i=1}^{I} w_{j i} x_{\min }\left(\Delta \psi+t^{*}\right)}{2 \Delta \psi}
\end{array}\right.
\end{gathered}
$$

\section{Simulation example}

The experiment environment: Pentium IV 2.4 GHz CPU, 4GB DDR RAM, 80GB+7200 RPM hard drive, Windows 7 operating system. In the experiment, the fault samples of analog circuit can be collected using Capture CIS module in software ORCAD 10.5. All simulations for the algorithms involved were implemented in MATLAB programming environment.

In the experiment, Sallen-Key bandpass filter is selected as the research subject of fault diagnosis for analog circuit. The nominal value of the components in the circuit is shown in Fig. 2. The normal tolerance range of resistance is set as $5 \%$. The normal tolerance range of capacitance is set as $10 \%$. The output "Out" is only test node. To simplify the problem, the paper only studies the single tolerance fault of resistance and capacitance without regard for multiple faults and hard faults.

The software ORCAD is used to simulate the circuit. Through AC small-signal sensitivity analysis, the sensitivity of the effect of each component on output waveform is compared comprehensively. It can be known that the change of $\mathrm{R}_{2}, \mathrm{R}_{3}, \mathrm{C}_{1}, \mathrm{C}_{2}$ has the greatest impact on the output response waveform of the circuit. Therefore, it can determine that the fault set contains 8 fault modes, namely $\mathrm{R}_{2^{-}}, \mathrm{R}_{2}+, \mathrm{R}_{3^{-}}, \mathrm{R}_{3}+, \mathrm{C}_{1^{-}}, \mathrm{C}_{1}+, \mathrm{C}_{2^{-}}, \mathrm{C}_{2}+$, where the symbol '+' and '-' represent too large tolerance fault and too small tolerance fault respectively. All nominal value of deviation error are set as $\pm 50 \%$. In this way, 8 fault modes together with normal mode add up to 9 modes. In addition, "0-l" method can be used to obtain the expected output vector of 8 output nodes in AUKF-WNN.

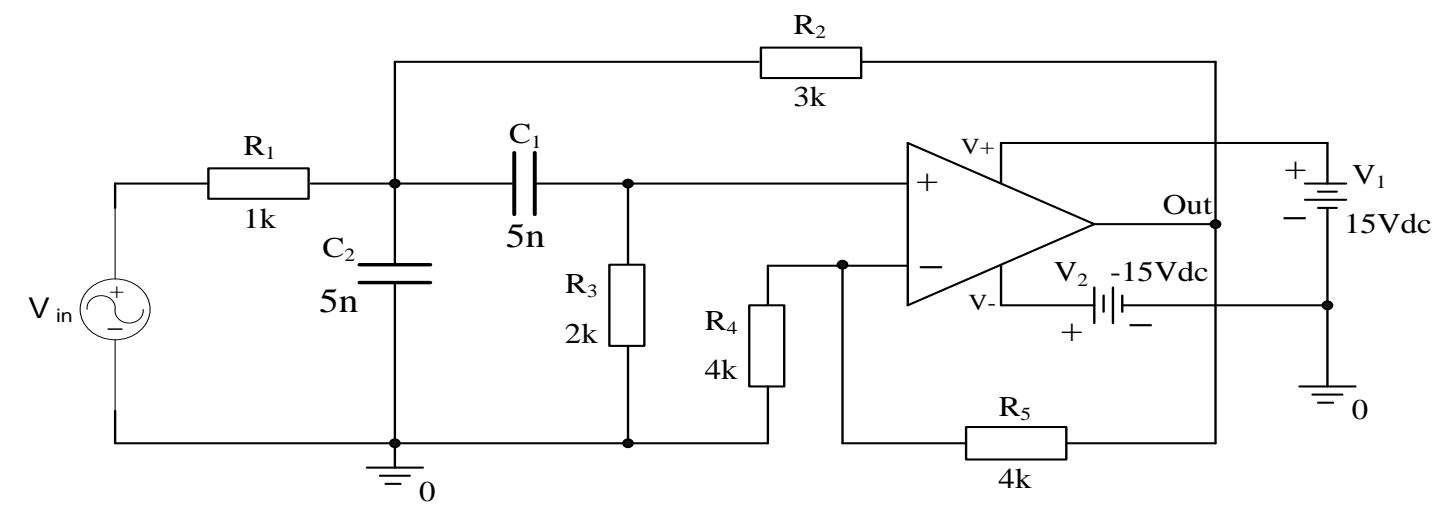

Fig. 2 Sallen-Key bandpass filter 
In order to verify the effectiveness of the proposed method, BP-WNN needs to be compared with BP-NN, RBF-NN, PSO-NN in fault diagnosis test for the selected circuit. The initial values of the parameters $w_{j i}, v_{k j}, a_{j}, b_{j}$ can be taken as the random number in [0,1]. Maximum number of training can be set as 500 . The wavelet basis function in the hidden layers of WNN can be selected as Morlet wavelet function $\psi(t)=\cos (1.75 t) e^{-t^{2} / 2}$. In the experiment, if the absolute value of difference between expected output and actual output of an output node in WNN is greater than the determining value 0.3 , then it can be considered that the diagnosis result is incorrect. The modeling capabilities of 4 methods are compared in Table 1 under different hidden nodes number, where IDN is incorrect diagnosis number, CIN is convergence iteration number.

Table 1 Comparison of modeling capability based on 4 methods

\begin{tabular}{ccccc}
\hline $\begin{array}{c}\text { Hidden nodes } \\
\text { number }\end{array}$ & $\begin{array}{c}\text { Diagnosis } \\
\text { model }\end{array}$ & $\begin{array}{c}\text { Training } \\
\text { IDN }\end{array}$ & $\begin{array}{c}\text { Test } \\
\text { IDN }\end{array}$ & CIN \\
\hline \multirow{2}{*}{10} & BP-NN & 37 & 52 & 500 \\
& RBF-NN & 19 & 34 & 500 \\
& PSO-NN & 28 & 37 & 500 \\
& BP-WNN & 21 & 28 & 478 \\
\hline \multirow{2}{*}{15} & BP-NN & 35 & 42 & 500 \\
& RBF-NN & 20 & 37 & 500 \\
& PSO-NN & 26 & 35 & 500 \\
& BP-WNN & 22 & 25 & 334 \\
\hline \multirow{2}{*}{20} & BP-NN & 45 & 64 & 500 \\
& RBF-NN & 23 & 35 & 500 \\
& PSO-NN & 32 & 43 & 500 \\
& BP-WNN & 25 & 31 & 428 \\
\hline
\end{tabular}

\section{Conclusions}

To improve the diagnosis accuracy of tolerance fault for analog circuit, a WNN tolerance fault diagnosis method is proposed on the basis of parameter initialization. The simulation on Sallen-Key bandpass filter shows that, WNN diagnosis model has fast convergence and high accuracy, greatly improving the diagnosis performance of tolerance fault.

\section{References}

[1] R. S. Berkowitz. Condition for network-element-value solvability. IRE Transaction on Circuit Theory, 15(9), 1962, 25-29

[2] N. Navid, A. A. Willson. A theory and an algorithm for analog circuit fault diagnosis. IEEE Transaction on CAS, 26(7), 1979, 440-457

[3] Y. H. Tan, Y. G. He. Wavelet method for fault diagnosis of analogue circuits. Transactions of China Electro-technical Society, 20(8), 2005, 89-93

[4] M. F. Peng, Y. G. He, Y. N. Wang, et al. Synthetic intelligence based fault diagnosis of analog circilts. Proceedings of the CSEEE, 26(3), 2006, 19-24

[5] P. P. Preseren, B. Stopar. Wavelet neural network employment for continuous GNSS orbit function construction: application for the assisted-GNSS principle. Applied Soft Computing, 13(5), 
2013, 2526-2536

[6] O. Yacine, D. Gerard. Initialization by selection for wavelet network training. Neurocomputing, 34, 2000, 131-143. 